and considering the scale of peptide size versus the structure of the peptide-binding site, with its raised $\alpha$-helical walls, intimate contact between the TCR and peptide probably necessitates simultaneous TCR-MHC interaction ${ }^{12.13}$. Furthermore, there is indirect evidence that during antigen recognition TCRs do make specific MHC contacts that are influenced by, or directly involve, allelically polymorphic residues ${ }^{7.14-17}$. To accomplish the optimal diversification of the peptide-binding site, in some cases alterations in regions simultaneously accessible to both the TCR and peptide could have been selected; as a result, during peptide recognition, TCR-MHC interactions occur that need to take this allele-specific structural variation into account. To achieve the selection of receptors that can meet this requirement, TCRs are 'pre-tested' on self peptides bound to the presenting molecules available. Selection for TCRs able to bind self peptide presented by a given $\mathrm{MHC}$ molecule will pari passu involve selection for adaptation to these allelically variable features of MHC molecules. This accommodation to self-MHC polymorphism does not need to contribute in a positive sense to the molecular interactions ${ }^{13}$. Rather, the receptor must prevent the unique allelic features of the self-MHC molecule from interfering with recognition of the bound peptide (self or foreign). This is an inversion of the usual thinking about thymic selection. Allelic polymorphism of MHC molecules is now seen to make its main contribution to selection by choosing peptides for presentation, and the principal binding event involves peptide recognition, not affinity for allelespecific MHC structure itself. In this view, T-cell bias towards self-MHC structure is merely a by-product of selection for recognition of self peptides bound by those MHC molecules. The self-peptides thus act as 'stand-ins' (structurally related analogues) for the unknown set of foreign peptides to be presented to mature T cells. MHC restriction would thus involve differences in the set of bound peptides that can be presented and the interfering effects of non-self MHC polymorphism on TCRs binding to peptide-MHC complexes.

Taking the crystallographic data ${ }^{2}$ on the existence of peptide-binding subsites into account, this model suggests that the spatial relationship of the site of TCRself-peptide contact (epitope) to the sites of TCR-MHC contact is constrained by the subsite into which the peptide binds. This leads to the selection of a set of TCRs that are accommodated to the $\mathrm{MHC}$ molecule in an orientation which, for effective recognition, requires that the epitopes of foreign peptides must be similarly located with respect to the overall MHC-binding site. This can be accom-

\title{
Quasicrystals turn to the sixth-dimension
}

LOOKING at the shadow of a thin slice of a simple cubic lattice you will see, depending on the lattice's orientation, a square or a hexagonal array. Or you may even see a seemingly disordered pattern of dots, although in fact along each of two directions, the lattice points will be arranged according to two incommensurate lattice spacings. Quasicrystals, such as discovered by Shechtman et al. ${ }^{1}$ in 1984, exhibit a similar type of three-dimensional disorder, and can also have incommensurate lattice steps in the same direction.

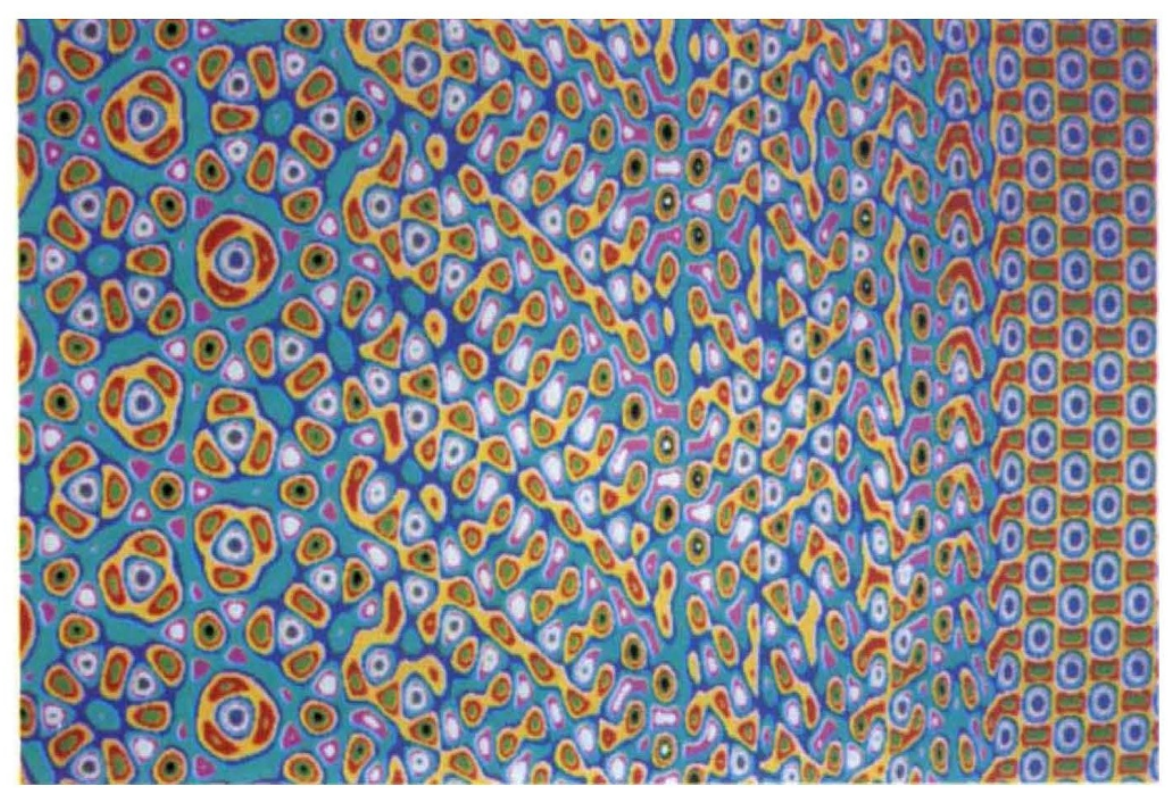

By analogy with the cubic-lattice example, this suggests that quasicrystals can be regarded as three-dimensional projections or 'shadows' of a six-dimensional regular lattice. As with the cubiclattice case, the projection can be rotated to give a different view ${ }^{2}$. Indeed, the concept goes back at least to Coxeter's shadows of parallelotopes ${ }^{3}$.

Torres, Pastor and Jiménez, using algorithms developed to study quasicrystals ${ }^{4,5}$, have drawn a composite view of a three-dimensional slice of a sixdimensional 'hypercubic' lattice, with the projection orientation varied continuously

plished in most cases only by binding the foreign peptide to the same subsite, based on a structural similarity with the MHCinteracting portion of the self peptide. In the example reported by Nikolić-Žugić a change in or loss of a given binding pocket in the $\mathbf{K}^{\mathrm{hmg}}$ molecule presumably eliminates the presentation of a subset of self peptides whose epitopes guide the selection of certain TCRs, specifically those able to see the OVA peptide bound to this same subsite in the wild-type $\mathrm{K}^{\mathrm{b}}$ molecule. The alteration of this binding pocket would also account for the failure of the from left to right across the picture. At one end, we look along an axis with fivefold symmetry to see an incommensurate quasilattice; at the other, we look along a fourfold direction to see a rational lattice. In between, the angle of view changes. The picture thus represents a kind of interface between quasicrystalline and truly crystalline phases and as such might be compared with actual micrographs of grain boundaries.

The authors have compared the picture to the well known Metamorphosis print by
M.C. Escher. It is a kind of Bloch wall, rotating with one parameter from one structure to another across a boundary of finite thickness.

ALAN MACKAY

Alan Mackay is in the Department of Crystallography, Birkbeck College, London WC1E $7 H X, U K$.

1. Shechtman, D. et al. Phys. Rev. Lett. 53, 1951-1953 (1984)

2. Kramer, P. \& Neri, R. Acta Cryst. A40, 580-587 (1984)

3. Coxeter, H.S.M. Regular Polytopes (Macmillan, London 1948)

Torres, M., Pastor, G. \& Jiménez, J. J. Phys. (Cond Matter) 1, 6877-6880 (1989)

5. Torres, M., Pastor, G. \& Jiménez, J. Phil. Mag. Lett. 59 , 181-188 (1989)

$\mathrm{K}^{\mathrm{hms}}$ molecule to present the OVA peptide to OVA-K ${ }^{\mathrm{b}}$-specific $\mathrm{T}$ cells.

This model has a general and testable prediction - individual mutations of a given MHC molecule in TCR-inaccessible locations affecting the binding of distinct subsets of foreign peptides will have similar selective effects on the binding of self peptides. This in turn should lead to predictable effects on intrathymic positive selection of the repertoire for these same foreign peptides. The analysis of antigen responses by transgenic mice produced with such mutant MHC molecules pro- 